\title{
Structural Relaxation in Silicate Melts and Non-Newtonian Melt Rheology in Geologic Processes
}

\author{
Donald B. Dingwell and Sharon L. Webb \\ Bayerisches Geoinstitut, Universität Bayreuth, Postfach 101251, 8580 Bayreuth, West Germany
}

Dedicated to the memory of Chris Scarfe

\begin{abstract}
The timescale of structural relaxation in a silicate melt defines the transition from liquid (relaxed) to glassy (unrelaxed) behavior. Structural relaxation in silicate melts can be described by a relaxation time, $\tau$, consistent with the observation that the timescales of both volume and shear relaxation are of the same order of magnitude. The onset of significantly unrelaxed behavior occurs $2 \log _{10}$ units of time above $\tau$. In the case of shear relaxation, the relaxation time can be quantified using the Maxwell relationship for a viscoelastic material; $\tau_{\mathrm{S}}=\eta_{\mathrm{S}} / G_{\infty}$ (where $\tau_{\mathrm{S}}$ is the shear relaxation time, $G_{\infty}$ is the shear modulus at infinite frequency and $\eta_{\mathrm{S}}$ is the zero frequency shear viscosity). The value of $G_{\infty}$ is known for $\mathrm{SiO}_{2}$ and several other silicate glasses. The shear modulus, $G_{\infty}$, and the bulk modulus, $K_{\infty}$, are similar in magnitude for every glass, with both moduli being relatively insensitive to changes in temperature and composition. In contrast, the shear viscosity of silicate melts ranges over at least ten orders of magnitude, with composition at fixed temperature, and with temperature at fixed composition. Therefore, relative to $\eta_{\mathrm{s}}, G_{\infty}$ may be considered a constant (independent of composition and temperature) and the value of $\tau_{\mathrm{S}}$, the relaxation time, may be estimated directly for the large number of silicate melts for which the shear viscosity is known.

For silicate melts, the relaxation times calculated from the Maxwell relationship agree well with available data for the onset of the frequency-dependence (dispersion) of acoustic velocities, the onset of non-Newtonian viscosities, the scan-rate dependence of the calorimetric glass transition, with the timescale of an oxygen diffusive jump and with the $\mathrm{Si}-\mathrm{O}$ bond exchange frequency obtained from ${ }^{29}$ Si NMR studies.

Using data obtained over a range of frequencies and strain-rates we illustrate the significance of relaxed versus unrelaxed behavior in laboratory experiments on silicate melts. Similarly, using strain-rate estimates for magmatic processes we evaluate the significance of the liquid-glass transition in igneous petrogenesis.
\end{abstract}

\section{Introduction}

There now exist a number of studies of physical properties of silicate melts that demonstrate a frequency- or strain-rate dependence of the observed property (e.g., acoustic velocities, Laberge et al. 1973, Sato and Manghnani 1985, Rivers and Carmichael 1987: viscosity, Li and Uhlmann 1970, Simmons et al. 1982). In these studies the timescale of relaxation (or response) of the silicate melt structure is similar in magnitude to, or longer than, the timescale of observation and the observed properties correspond to an unrelaxed state. In addition, there is a wealth of data on the relaxed properties of silicate melts (e.g. shear viscosity, Ryan and Blevins 1987; density, Lange and Carmichael 1987). The key to predicting relaxed and unrelaxed behavior in silicate melts is the determination of the liquid-glass transition in time-temperature space for each silicate melt composition.

This paper emphasizes that all the available evidence from a variety of experimental sources (e.g. shock wave, ultrasonic, fiber elongation, torsional testing, concentric cylinder viscometry, scanning calorimetry, ${ }^{29} \mathrm{Si}$ NMR) is consistent with the description of the liquid-glass transition as a curve in time-temperature space, whose location can be predicted from shear viscosity data for each composition.

\section{The Glass Transition}

The liquid to glass transition occurs when the liquid cannot achieve an equilibrium response to an external perturbation of its state (e.g., a variation in temperature, stress, electric, or magnetic field). For example, the glass transition is observed in viscometry when the timescale of the liquid response (or relaxation) becomes longer than the timescale of observation (defined as the inverse of the strain-rate). Correspondingly, the glass transition is observed in ultrasonic measurements of melt properties when the angular frequency of the acoustic wave becomes greater than the inverse of the relaxation time.

The glass transition is often described by a single characteristic temperature $\left(T_{\mathrm{g}}\right)$ which is termed the glass transition temperature. Defining the glass transition as a single temperature follows from the fact that most laboratory methods used to measure the glass transition (e.g., scanning calorimetry, dilatometry) are usually performed on a laboratory timescale of several seconds to several minutes. Therefore, the time available for the relaxation of the material of interest in response to the applied perturbation is essentially constant. More generally, the glass transition is described by a curve in temperature-time space with increasing temperature reducing the time required for structural relaxation (e.g., Tauke et al. 1968). For each silicate melt composition, the relaxation time curve divides temperature-time space into two regions; the liquid field (long timescales and high 


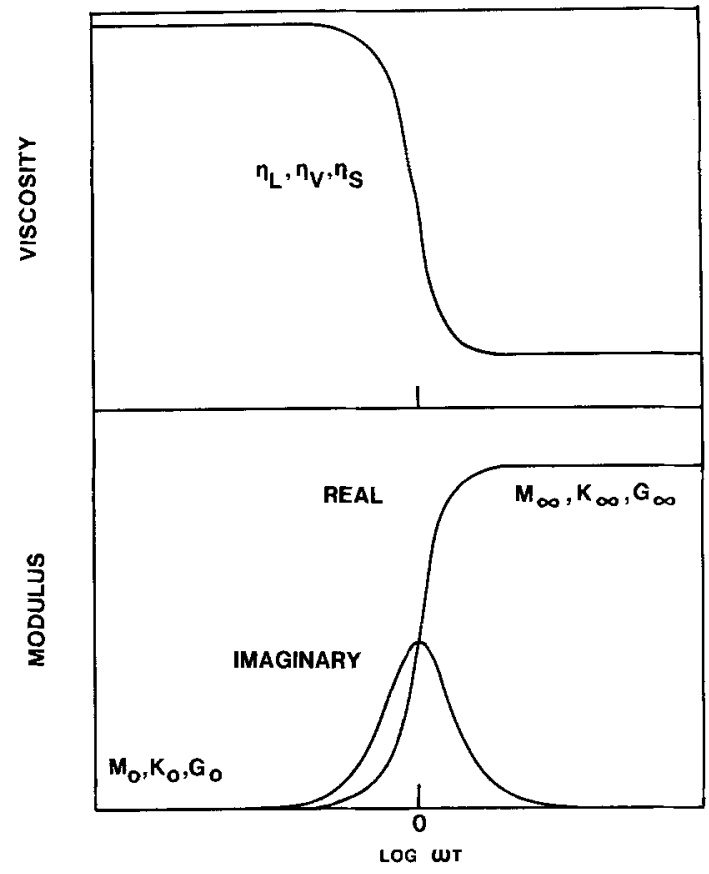

Fig. 1. Calculated frequency-dependent behavior of longitudinal, volume and shear viscosities $\left(\eta_{\mathrm{L}}, \eta_{\mathrm{V}}\right.$ and $\eta_{\mathrm{S}}$, respectively) and moduli ( $M, K$ and $G$, respectively) of a viscoelastic material with a relaxation time, $\tau$, plotted as a function of $\omega \tau$. The subscripts " 0 " and " $\infty$ " indicate zero frequency and infinite frequency values

temperatures) and the glass field (short timescales and low temperatures). At any given temperature, rates of perturbation faster than the rate of structural relaxation lead to glassy or solid-like responses, while rates of perturbation slower than that of structural relaxation lead to liquid-like responses of the material.

\section{Structural Relaxation}

The response of a material to each perturbation will have its own relaxation (equilibration) timescale. Different structural components of a material will also respond to the same perturbation on differing timescales. Therefore a glass transition may be described for every perturbation and its associated property. This paper is confined to the discussion of the slowest structural relaxation in liquids in response to changes in the stress field. In general, a change in the stress field will result in a combination of volume and shear relaxation, but below we show that the timescales of both volume and shear relaxation are similar in silicate liquids.

Figure 1 illustrates the frequency-dependent modulus and viscosity calculated for a viscoelastic material, with relaxation time $\tau$, in response to the application of a sinusoidal perturbation (e.g., an acoustic wave). The observed modulus of a material is determined as the ratio of stress $\sigma$ to strain $\varepsilon$ (Nye 1957). For a viscoelastic material, the strain due to the application of a constant stress has contributions from both elastic and viscous properties (Jackson 1986);

$\frac{\varepsilon(t)}{\sigma}=\left(M^{-1}+t / \eta\right)$

for the elastic modulus $M$, Newtonian viscosity $\eta$, and time $t$. For a sinusoidal stress $\sigma(\omega)=\sigma_{0} \exp ^{i \omega t}$, the strain is (Nowick and Berry 1972, Jackson 1986);
$\frac{\varepsilon(\omega)}{\sigma(\omega)}=M^{-1}(1-i / \omega \tau)$

and the observed modulus is a function of the frequency of the applied stress (Herzfeld and Litovitz 1956);

$M^{*}(\omega)=\frac{\sigma(\omega)}{\varepsilon(\omega)}=M_{0}+\frac{M\left(\omega^{2} \tau^{2}+i \omega \tau\right)}{1+\omega^{2} \tau^{2}}=M^{\prime}(\omega)+i M^{\prime \prime}(\omega)$,

where $\omega(=2 \pi f)$ is the angular frequency of the stress wave, $M_{0}$ is the relaxed modulus of the material, $M_{0}+M=M_{\infty}$ is the unrelaxed modulus and $\tau$ is the relaxation time of the material. The modulus determined from the application of a sinusoidal perturbation has a real, $M^{\prime}(\omega)$, and an imaginary component, $M^{\prime \prime}(\omega)$. These components are commonly termed the storage and loss moduli, respectively (Mills 1974, Rosen 1982), as they describe the storage and the loss of energy from the applied perturbation to the material. From Equation 3, and Figure 1, it can be seen that the observed modulus approaches the value of the elastic (unrelaxed) modulus of the material when $\omega \tau \gg 1$.

The viscosity of a material is the ratio of the stress, $\sigma$, to the strain-rate, $\dot{\varepsilon}$. The viscosity of a viscoelastic material, determined by the application of a sinusoidal stress, is (Bird et al. 1977);

$\eta^{*}(\omega)=\frac{\sigma(\omega)}{\dot{\varepsilon}(\omega)}=\frac{M^{*}(\omega)}{i \omega}=\eta \cdot \frac{1-i \omega \tau}{1+\omega^{2} \tau^{2}}=\eta^{\prime}(\omega)+i \eta^{\prime \prime}(\omega)$

where $\eta$ is the Newtonian viscosity of the material. Again, it is seen that the observed viscosity is a function of the frequency of the perturbation. Analogous to the modulus, there exist loss, $\eta^{\prime}(\omega)$, and storage, $\eta^{\prime \prime}(\omega)$, viscosity terms. The imaginary parts of the modulus and viscosity are described by Debye functions (Nowick and Berry 1972). Therefore, the FWHM of the imaginary peak is $1.144 \mathrm{log}$ units in frequency or time space, and significant deviation $(2 \%)$ from relaxed behavior is expected to occur $2 \log$ units above the relaxation time $\tau=(2 \pi f)^{-1}$.

Equations 3 and 4 describe the frequency-dependence of the shear, longitudinal and volume viscosities and moduli. Given that the longitudinal modulus $M$, is a function of the shear and volume (bulk) moduli, $G$ and $K$, respectively (Nye 1957),

$M=K+4 G / 3$

the longitudinal viscosity is also a function of the shear and volume viscosities,

$\eta_{\mathrm{L}}=\eta_{\mathrm{V}}+4 \eta_{\mathrm{S}} / 3$.

For frequencies such that $\omega \tau \ll 1$, the viscous properties of the material dominate the observed stress/strain behavior, whereas for frequencies where $\omega \tau \gg 1$, the elastic properties of the material dominate the observed behavior. In the region where $\omega \tau \approx 1$, viscous and elastic behavior coexist and the attenuation coefficient, $\alpha$, of the perturbation travelling through the material at these frequencies can be determined (O'Connell and Budiansky 1978, Rivers and Carmichael 1987);

$\alpha \lambda \cong \frac{\pi M^{\prime \prime}(\omega)}{M^{\prime}(\omega)}=\frac{\pi M \omega \tau}{M_{0}+\left[M_{0}+M\right] \omega^{2} \tau^{2}}$.

If the relaxation of a liquid is described by a distribution of relaxation times, the width of the $M^{\prime \prime}(\omega)$ and $\alpha \lambda$ curves in $\omega \tau$ space will be larger than the Debye function width. 


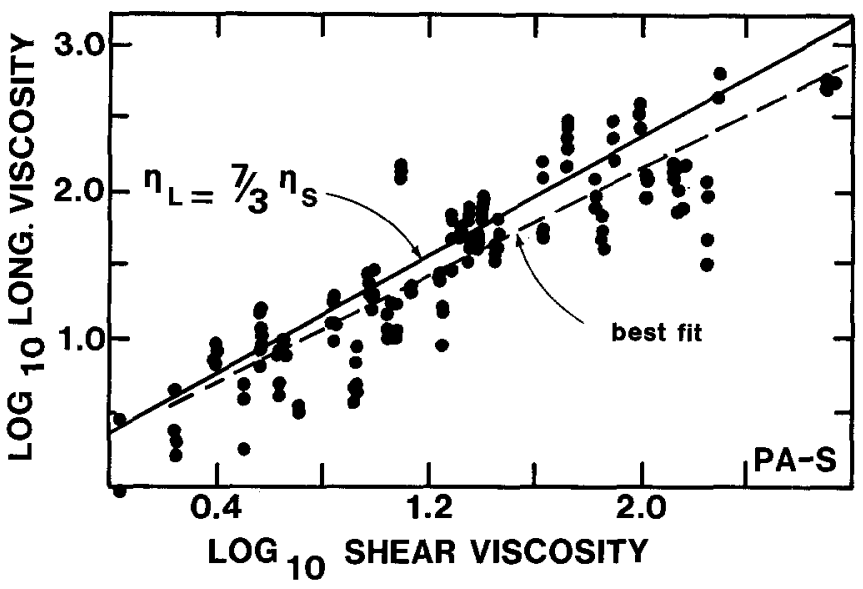

Fig. 2. The relationship between longitudinal and shear viscosities in silicate melts. The solid line represents the correlation predicted from the assumption that the volume and shear viscosities are equal. The dashed line is the best fit from a least squares regression and lies within $1 \sigma$ of the theoretical line. The longitudinal data are from Rivers and Carmichael (1987) and the shear data are from references therein

Similarly, the width of these curves will increase in the case of longitudinal measurements, should the shear and volume relaxation times not be equal.

\section{Volume Versus Shear Viscosity}

Volume and shear viscosity are measures of the resistance of the structure to volumetric and shear flow, respectively. The shear viscosity can be measured directly in a simple shear field involving no volume changes. This is commonly done for silicate melts using the technique of concentriccylinder viscometry, and a wealth of shear viscosity data has resulted from such studies (e.g. Ryan and Blevins 1987). The geometries of other common methods of viscosity determinations for silicate melts (including beam bending, micropenetration and fiber elongation) involve components of both volume and shear stress and thus the derived viscosity is a mixture of both the volume and shear viscosities.

The volume viscosity can be obtained directly from studies of volume relaxation of densified melts (e.g., Höfler and Seifert 1984) or of glasses with different cooling histories (Ritland 1954). Also, an extensive data set can be extracted from the ultrasonic data on silicate melts (e.g., Laberge et al. 1973, Rai et al. 1981, Sato and Manghnani 1985, Rivers and Carmichael 1987). Most such studies report the longitudinal viscosity and modulus of liquids. The volume viscosity of a silicate liquid can be calculated, using Equation 6, from the longitudinal viscosity data of ultrasonic studies in combination with the shear viscosity data of concentric cylinder viscosity studies. Such calculations by Sato and Manghnani (1985) resulted in the conclusion that their frequency-independent volume viscosities were similar in magnitude to the shear viscosities of their basalt melts.

Figure 2 is a plot of frequency-independent longitudinal viscosity against shear viscosity for silicate melts using the data set of Rivers and Carmichael (1987). Setting volume and shear viscosities to be equal, a line of slope 1 and intercept $\log _{10}(7 / 3)$ is predicted from Equation 6. As can be seen in Figure 2, the best fit line to this data has slope and intercept values within $1 \sigma$ error of the line predicted from Equation 6, indicating that volume and shear viscosit- ies are indeed equal. (The slightly low slope arises, at least in part, from the inclusion of all of Rivers and Carmichael's (1987) data which allow the calculation of $\eta_{\mathrm{v}}>0$. These results in the inclusion of slightly unrelaxed (underestimated) $\eta_{\mathrm{L}}$ values with increasing $\eta_{\mathrm{S}}$.)

\section{Relaxation Timescales}

The ratio of viscosity (volume or shear) to modulus (bulk or shear) gives the timescale of structural relaxation of a melt (Herzfeld and Litovitz 1956). The relaxation time relationship for viscoelastic materials is given by the Maxwell relation;

$\begin{aligned} \tau_{\mathrm{S}} & =\eta_{\mathrm{S}} / G_{\infty} \\ \tau_{\mathrm{V}} & =\eta_{\mathrm{V}} / K_{\infty}\end{aligned}$

where $\tau_{\mathrm{S}}$ and $\tau_{\mathrm{V}}$ are the relaxation times; $G_{\infty}$ and $K_{\infty}$ are the infinite frequency (unrelaxed) moduli and $\eta_{\mathrm{S}}$ and $\eta_{\mathrm{V}}$ are the relaxed viscosities, for shear and volume, respectively (Gruber and Litovitz 1964).

The values of $G_{\infty}$ and $K_{\infty}$, the rigidity and bulk modulus respectively, have been measured at room temperature for many silicate glasses and a useful compilation is provided by Bansal and Doremus (1986). $G_{\infty}$ ranges from 5 to $42 \mathrm{GPa}$ over a compositional range spanning 5-99 mole $\% \mathrm{SiO}_{2}$; while $K_{\infty}$ ranges from 25 to $78 \mathrm{GPa}$. Studies of the temperature dependence of these moduli are much more limited. Data are available for amorphous $\mathrm{SiO}_{2}$ (from 400 to $1600^{\circ} \mathrm{C}$ ) using Brillouin scattering techniques (Bucaro and Dardy 1974) in which the infinite frequency bulk modulus, $K_{\infty}=43 \mathrm{GPa}$, the shear modulus, $G_{\infty}=33 \mathrm{GPa}$ and the longitudinal modulus, $M_{\infty}=88 \mathrm{GPa}$. These moduli are relatively insensitive to temperature over this range (varying by less than $5 \%$ ).

$G_{\infty}$ data is also available from torsional measurements of storage and loss moduli (Kurkjian 1963 and references therein, Larsen et al. 1974, Mills 1974). For example, Mills (1974) directly measured the shear modulus of $\mathrm{Na}_{2} \mathrm{Si}_{2} \mathrm{O}_{5}$ composition in the temperature range of 400 to $500^{\circ} \mathrm{C}$ and the frequency range from $10^{-3}$ to $1 \mathrm{rad} \mathrm{s}^{-1}$, obtaining a value of $18 \pm 2 \mathrm{GPa}$ for $G_{\infty}$.

All the $\bar{G}_{\infty}$ and $K_{\infty}$ data available for silicate and nonsilicate glasses indicate that, compared with $\eta_{\mathrm{s}}$, the composition-dependence of $G_{\infty}$ and $K_{\infty}$ is relatively small (Gruber and Litovitz 1964, Litovitz and Davis 1965, Bucaro and Dardy 1974, Angell and Torell 1983, Bansal and Doremus 1986). This leads to the conclusion that $G_{\infty}$ and $K_{\infty}$ can be treated as constant relative to $\eta_{\mathrm{S}}$ and $\eta_{\mathrm{V}}$ in Equation 8 . The similarity in magnitude of the shear and bulk moduli and of shear and volume viscosities (Figure 2) result in the conclusion (from Equation 8) that the shear and volume relaxation times, $\tau_{\mathrm{S}}$ and $\tau_{\mathrm{V}}$, must also be similar in magnitude.

In the subsequent discussion we will use an average value of $\log _{10} G_{\infty}(\mathrm{GPa})=10 \pm 0.5$ (i.e., $G_{\infty}$ from 3.2 to $32 \mathrm{GPa}$ ) for all silicate melt compositions.

\section{Temperature- and Composition-Dependence of $\tau$}

One should not conclude from the above observation of the invariance of $G_{\infty}$ with respect to composition that the timescale of relaxation is independent of composition in silicate liquids. On the contrary, the abundant data on the large composition-dependence of shear viscosity allow us 


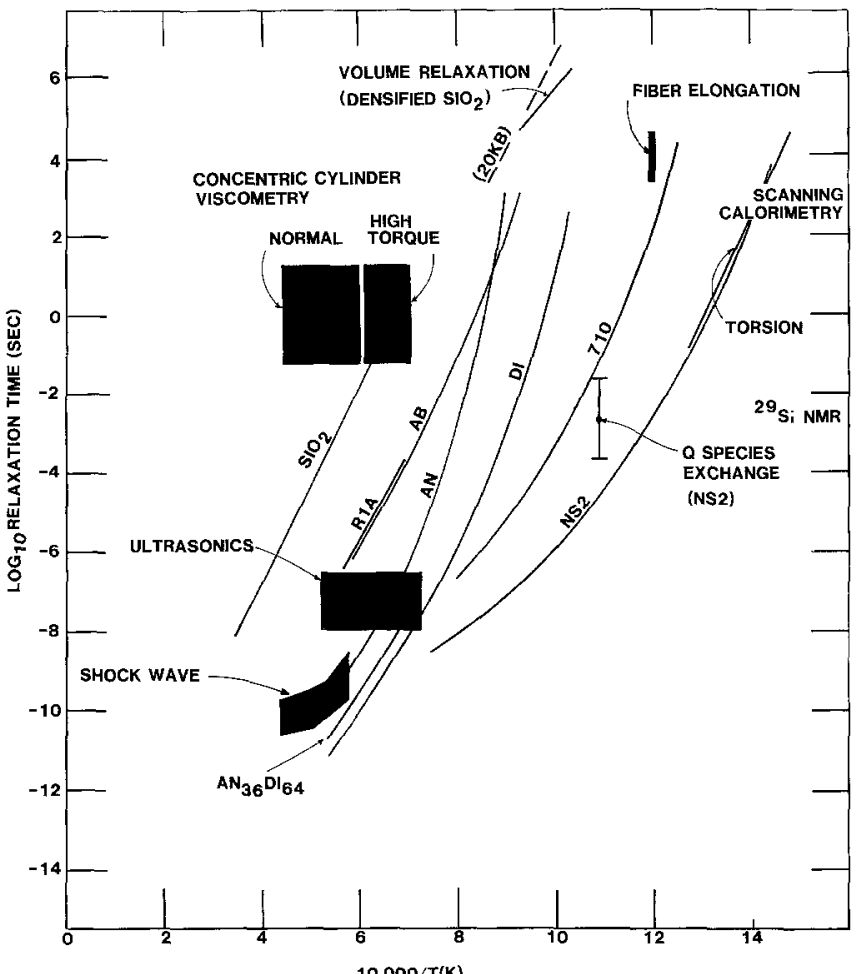

Fig. 3. The liquid-glass transition as a function of temperature plotted for several silicate melts calculated with Equation 8. The viscosity data used are from references in Richet (1984) for $\left(\mathrm{SiO}_{2}, \mathrm{AB}\right.$ albite, AN-anorthite, DI-diopside): Astin (1962: 710-soda lime SRM 710) and calculated using the method of Shaw (1972: R1A rhyolite). Other sources of data relating to relaxation times are discussed in the text, including volume relaxation of densified $\mathrm{SiO}_{2}$ (Höfler and Seifert 1984); fiber elongation (Simmons et al. 1982); high-temperature ${ }^{29} \mathrm{Si}$ NMR (Liu et al. 1988); normal (Dingwell, unpubl.) and high torque (Spera et al. 1988) concentric cylinder viscometry; ultrasonics (Sato and Manghnani 1985); shock wave (Rigden et al. 1988)

to demonstrate (via Equation 8 ) that $\tau_{\mathrm{s}}$ is a strong function of composition (and temperature). Figure 3 is a plot of calculated $\tau_{\mathrm{s}}$ versus reciprocal absolute temperature for a number of well-investigated silicate melts. These include $\mathrm{SiO}_{2}$, albite, anorthite, diopside, $\mathrm{Na}_{2} \mathrm{Si}_{2} \mathrm{O}_{5}$, and NBS standard soda-lime glass (SRM 710). As noted above, we estimate that $\log _{10} G_{\infty}(\mathrm{GPa})=10 \pm 0.5$ (this $0.5 \log$ unit variation in $G_{\infty}$ is much larger than the errors in viscosity ( $\sim 0.05 \log$ units ( $\mathrm{Pa} \mathrm{s})$ )). Also included in this figure are the ranges of some experimental determinations of melt properties. It can be seen in Figure 3 that the relaxation time for each silicate melt is a strong function of temperature. The compositional-dependence of relaxation times can be estimated from isothermal comparisons. Such comparisons, between the viscosity extremes of $\mathrm{SiO}_{2}$ and $\mathrm{Na}_{2} \mathrm{Si}_{2} \mathrm{O}_{5}$, illustrate that relaxation times vary over tens of orders of magnitude at very low temperatures (e.g. below $1000^{\circ} \mathrm{C}$ ), range over approximately 10 orders of magnitude at intermediate temperatures of 1100 to $1300^{\circ} \mathrm{C}$, and converge at higher temperatures.

The $\tau_{\mathrm{s}}$ values of Mills (1974) are shown in Figure 3 as a solid line labelled "torsion". The data fall on the $\tau$ curve calculated for $\mathrm{Na}_{2} \mathrm{Si}_{2} \mathrm{O}_{5}$ from shear viscosity data, providing independent confirmation of our estimate of the position of the $\tau$ curve for $\mathrm{Na}_{2} \mathrm{Si}_{2} \mathrm{O}_{5}$.

\section{The Viscoelastic Response}

The imaginary part of the complex longitudinal modulus determined in ultrasonic studies is due to the phase lag of the response of a viscoelastic material to the perturbing stress field in the region where both viscous (relaxed) and elastic (unrelaxed) properties are observed. This coexistence of significant viscous and elastic behavior is viscoelastic response (Rosen 1982). The maximum of the imaginary modulus, $M^{\prime \prime}$, and of the attenuation curve, $\alpha \lambda$, in the frequency domain is a measure of the single or the most probable relaxation time $\left(\tau=1 / 2 \pi f_{\max }\right)$ for the melt. The width of the curve is a measure of the region in the frequency (or time) domain in which significant viscoelastic behavior is observed (Herzfeld and Litovitz 1956).

There are several torsional studies which record the onset of viscoelastic response (i.e. the imaginary part of the complex shear modulus) $2 \log _{10}$ units of time above the relaxation time (Kurkjian 1963, Mills 1974, Larsen et al. 1974). Figure 11 of Rivers and Carmichael (1987) is a plot of longitudinal $\alpha \lambda$ values for a wide range of silicate melts. Rivers and Carmichael (1987) fitted the $\alpha \lambda$ values of their 32 silicate melt compositions at all temperatures and frequencies of measurement with a function (Equation 7) consistent with the approximation of a single relaxation time for longitudinal relaxation (i.e., $\tau_{\mathrm{S}}=\tau_{\mathrm{V}}=\tau_{\mathrm{L}}$; Rivers and Carmichael 1987). In Figure 3, this also corresponds to the onset of significant unrelaxed behavior approximately $2 \log _{10}$ units of time above the $\tau$ curve for each composition. The onset of non-Newtonian theology is therefore expected to occur $2 \log _{10}$ units of time above the $\tau$ curves of Figure 3 .

\section{Newtonian Versus Non-Newtonian Rheology}

The relaxation time-temperature relationships obtained assuming a constant value of $G_{\infty}$ (illustrated in Figure 3 ) are consistent with two well-documented cases of non-Newtonian viscosity in silicate melts (Li and Uhlmann 1970, Simmons et al. 1982) and with the much more common observation of Newtonian viscosity.

The key to reconciling Newtonian and non-Newtonian viscosity data for silicate melts lies in the shear rate-temperature location of the viscosity experiments with respect to the relaxation time curve for the melt composition. In Figure 3 we have plotted (as boxes) the timescale (strain-rate ${ }^{-1}$ ) and temperature ranges of some experimental studies of silicate melt viscosity.

The most widely used method of silicate melt shear viscosity determination is the concentric cylinder method. In concentric cylinder viscometry, the maximum torque and the minimum rotation speed define the maximum viscosity measurable. On most commercial viscometers (e.g. Brookfield ${ }^{\circledR}$ Engineering) the limits are approximately 5 $\times 10^{-3} \mathrm{Nt} \mathrm{m}$ and $0.5 \mathrm{rpm}$, respectively. These limits result in a maximum measurable viscosity of approximately $10^{5} \mathrm{~Pa}$ s. Using Equation 8 and the maximum rotation speed $(200 \mathrm{rpm})$ of such viscometers we calculate the minimum viscosity at which non-Newtonian behavior should be observable to be $11.3 \pm 0.5 \log _{10}$ units (Pa s). This viscosity is well above the range of such viscometers. This illustrates that the viscosity-strain rate regime of most concentric cylinder viscometry (Figure 3 ) lies well above the liquidglass transition for all liquid silicates measurable with such instruments. This is consistent with the reports of Newton- 
ian behavior in studies using these instruments (e.g., Scarfe et al. 1983, Dingwell et al. 1985).

To our knowledge, there are three studies reporting nonNewtonian behavior for silicate melts ( $\mathrm{Li}$ and Uhlmann

mann (1970) were the first to observe non-Newtonian behavior in silicate melts. They performed fiber elongation experiments on a $\mathrm{Rb}_{2} \mathrm{O}-\mathrm{SiO}_{2}$ glass with 8 mole percent $\mathrm{Rb}_{2} \mathrm{O}$. They recorded the onset of non-Newtonian behavior as a function of strain-rate at several temperatures between 480 and $550^{\circ} \mathrm{C}$. The data of Li and Uhlmann (1970) indicate the onset of non-Newtonian behavior at $2 \log _{10}$ units of time above the calculated $\tau$ curve for this composition.

Simmons et al. (1982) performed fiber elongation experiments on SRM 710 soda-lime glass using strain rates corresponding to a timescale of $10^{2.85}$ to $10^{4.44} \mathrm{~s}$. In this study, only non-Newtonian behavior was observed (as a decrease in viscosity with strain-rate). The upper limit of their timescale yields values of viscosity within $0.1 \log _{10}$ units of the well-known relaxed viscosity (Astin 1962). The close approach of the Simmons et al. (1982) long-timescale data to the relaxed value for viscosity suggests these measurements were in the immediate vicinity of the onset of non-Newtonian behavior. Their position with respect to the $\tau$ curve for SRM 710 soda-lime glass is illustrated by a box labelled "fiber elongation" in Figure 3. The low strain-rate limit of their experiments lies $1.9 \log _{10}$ units of time above the $\tau$ curve.

The third study of interest is that of Spera et al. (1988) on rhyolite composition melts. Their study was performed with a high torque concentric-cylinder viscometer on two rhyolite melts with 3 to 8 volume percent air bubbles. In Figure 3 we have plotted (using relaxed viscosity calculated from the method of Shaw (1972)) the $\tau$ curve of Spera et al.'s (1988) R1A rhyolite. We have also plotted the location of these experiments in relaxation time-temperature space (labelled "high torque"). It can be seen that Spera et al.'s (1988) data were obtained at $\tau$ values from 2 to $6 \log _{10}$ units of time above the calculated $\tau$ curve. Thus, from the present analysis, it appears unlikely that Spera et al. (1988) were observing unrelaxed melt behavior.

\section{Microscopic Insights}

The self diffusivity of oxygen in silicate melts has been correlated with shear viscosity through the Stokes-Einstein and Eyring equations (Glasstone et al. 1941, Dunn 1982, Shimi$\mathrm{zu}$ and Kushiro 1984). The Eyring equation is:

$D=\mathrm{k} T / \eta \lambda$

for $D, \mathrm{k}, T, \eta$ and $\lambda$, the oxygen diffusivity, Boltzmann's constant, temperature (K), viscosity and the diffusive jump length (which may be taken as the diameter of an oxygen anion), respectively. The viscosity required in Equation 9 is that experienced by a diffusing particle in a direction perpendicular to the diffusion front (i.e., a diffusive jump). Such a strain is is equivalent to the strain induced by a longitudinal wave and the viscosity appropriate to Equation 9 is longitudinal viscosity. From Figure 2 we set longitudinal viscosity, $\eta_{\mathrm{L}}=(7 / 3) \eta_{\mathrm{S}}$.

Shimizu and Kushiro (1984) have plotted shear viscosity versus oxygen diffusivity for a number of melt compositions. Their Figure 2 indicates that viscosities obtained from the Eyring equation are overestimates of the shear viscosities.
A best fit line through the data yields calculated viscosities that are close to the Eyring prediction for an oxygen anion as the diffusing unit but are systematically low by a factor of 1.5 to 2.7 . If the longitudinal viscosity $\left(2.33 \eta_{\mathrm{s}}\right)$ is substituted for shear viscosity $\left(\eta_{\mathrm{s}}\right)$ in the comparison of Shimizu and Kushiro (1984) then the data lie within error $\left(0.2 \log _{10}\right.$ units) of the prediction of Equation 9, using a diffusing species diameter of $2.8 \times 10^{-10} \mathrm{~m}$. This success of the Eyring equation supports the proposal that the basic unit of viscous flow is the diffusive jump of $\mathrm{O}^{2-}$.

The Eyring equation appears to fail for $\mathrm{SiO}_{2}$. The calculated diffusivities for the $\mathrm{Si}-\mathrm{O}$ bond breaking mechanism using Equation 9 (viscosity data from Hofmaier and Urbain 1968) are approximately 3 orders of magnitude slower than oxygen diffusivities measured using gas permeation or exchange methods (Sucov 1963, Williams 1965, Muehlenbachs and Schaeffer 1977). This discrepancy may be explained by the proposal (e.g., Muehlenbachs and Schaeffer 1977) that the diffusion process being observed in these experiments involves transport of dissolved $\mathrm{O}_{2}$ gas (presumably as a neutral species). This interpretation is consistent with the low activation energy $\left(\sim 20 \mathrm{kcal} \mathrm{mole}^{-1}\right)$ for the process (Muehlenbachs and Schaeffer 1977). In contrast, the molecular dynamic experiments of Woodcock et al. (1976) indicate that the Eyring formalism is successful in predicting the oxygen diffusivities obtained in their work. Thus it is quite possible that the Eyring equation will predict the timescale of $\mathrm{O}$ diffusivity in the absence of excess $\mathrm{O}_{2}$ gas. Glass diffusion couple experiments for $\mathrm{SiO}_{2}$ (in vacuum) might resolve this question.

Additional evidence relating to the basic viscous flow process comes from high-temperature static ${ }^{29} \mathrm{Si} \mathrm{NMR}$ work of Liu et al. (1988). Liu et al. (1988) studied the static ${ }^{29} \mathrm{Si}$ NMR spectrum of $\mathrm{Na}_{2} \mathrm{Si}_{2} \mathrm{O}_{5}$ melt as a function of temperature. At low temperature they observed a distribution of $\mathrm{Q}$ species including $\mathrm{Q}_{2}, \mathrm{Q}_{3}$ and $\mathrm{Q}_{4}$. At temperatures near $650^{\circ} \mathrm{C}$ the distinction of $\mathrm{Q}$ species vanished and a single time-averaged position of the $\mathrm{Q}$ species, defined by the melt stoichiometry, was obtained. At temperatures near the loss of resolution of the Q species, Liu et al. (1988) have used the resonant frequency difference between $Q$ species as an estimate of the rate of exchange. Using a temperature of $648^{\circ} \mathrm{C}$ (from Figure 2 of Liu et al. 1988), we have plotted the point for their estimate of the timescale of $\mathrm{Q}$ species exchange in Figure 3. It falls above the $\tau$ curve for $\mathrm{Na}_{2} \mathrm{Si}_{2} \mathrm{O}_{5}$ but at the extremes of the estimated errors. This is consistent with the idea that the exchange of oxygens between Si atoms (involving breakage of the strongest bonds in the melt) is the origin of the slowest (i.e. structural) relaxation or glass transition. More recent, better data and lineshape simulations on $\mathrm{K}_{2} \mathrm{Si}_{4} \mathrm{O}_{9}$ liquid indicate that the species exchange rate is close to or slightly lower than the shear relaxation rate as estimated here; the apparent activation energy for the two processes is nearly identical (J. Stebbins, pers. comm.).

A final note of interest with respect to the atomistic significance of $\tau$ concerns the zero intercept values of the $\tau$ curves. If, as it appears in Figure 3, the $\tau$ curves are "compensated" (i.e. they have a common, $T^{-1}=0$, intercept value) then we are forced to seek an atomistic explanation for the infinite temperature value of $\tau$. Angell (1984) viewed the high temperature limit of $\tau$ as the case of the viscosity (and diffusion) timescale collapsing to the timescale of "vibration of the liquid quasi-lattice". The viscosities corre- 
sponding to this infinite temperature time scale of $10^{-14} \mathrm{~s}$ are $10^{-3.5}$ to $10^{-4.5} \mathrm{~Pa} \mathrm{~s}$. This is the range of viscosities of all gases (at all temperatures, Weast et al. 1972). Thus, in a sense, the gaseous state can be considered the limit of fragile liquids.

\section{High Frequency Spectroscopies and Experiments}

The $\tau$ curves for silicate melts illustrated in Figure 3 mark the onset of motional or jump diffusional line-broadening in any spectroscopic resolution of species defined by $\mathrm{Si}-\mathrm{O}$ bonds in silicate melts. The exact temperature for a given melt composition is derived from a knowledge of the timescale that corresponds to the particular radiation frequency or relaxation time of the spectroscopy being employed. Frequency scales are illustrated for several spectroscopies by Calas and Hawthorne (1988). Comparison of their Figure 3 with the present Figure 3 indicates that Raman, infrared and $\mathrm{X}$-ray spectroscopies will observe $\mathrm{Si}-\mathrm{O}$ bond motional averaging only at very high temperatures, for all melts. In contrast, spin-spin relaxation in NMR spectroscopy will produce lineshape coalescence resulting from motional averaging at frequencies corresponding to much lower temperatures (Lambert et al. 1981). We reiterate here that such a temperature limit is defined by the spectroscopy and the sample.

Ultrasonic wave propagation studies are also conducted at very high frequencies. Workers in ultrasonics are quite familiar with the onset of frequency-dependent properties and we have already referred to these studies briefly above. The general applicability of the Maxwell relation (Equation 8) is reinforced by the ultrasonic measurements of Rivers and Carmichael (1987). They report, however, that the most probable relaxation time is equal to $0.01 \eta_{\mathrm{s}} \beta$ (where $\beta$ is the relaxed compressibility). The zero frequency compressibility in this parameterization is substituting for $K_{\infty}^{-1}$ in the Maxwell relation. The factor of 0.01 in their equation is an error introduced by the fact that all the zero frequency shear viscosity data reported in Table 5 of Rivers and Carmichael (1987) are two orders of magnitude too large compared with the original references.

\section{High Pressure Studies}

Rigden et al. (1988) have performed shock wave studies on an anorthite-diopside, $1 \mathrm{~atm}$ eutectic melt composition $\left(\mathrm{An}_{36} \mathrm{Di}_{64}\right)$. In their paper they discuss the significance of the liquid-glass transition for their experiments. They calculate a characteristic time of the experiments, (the shock rise time), from the following

$\tau_{\mathrm{r}}=\frac{\eta U_{\mathrm{P}}}{U_{\mathrm{S}} \Delta P_{\mathrm{V}(\max )}}$

where $\tau_{\mathrm{r}}$ is the shock rise time, $\eta$ is the effective viscosity, $U_{\mathrm{s}}$ and $U_{\mathbf{P}}$ are the shock and particle velocities, and $P_{\mathrm{V}(\max )}$ is the maximum pressure offset between the equilibrium Hugoniot and the Rayleigh line (Rigden et al. 1988). In Figure 3 we have plotted the $\tau_{\mathrm{r}}$-temperature range calculated by Rigden et al. (1988) for their experiments and the $\tau_{\mathbf{S}}$ curve for the composition of their study, $\mathrm{An}_{36} \mathrm{Di}_{64}$, calculated from Equation 8.

Combining Equations 8 and 10 we obtain an expression for the ratio of $\tau_{\mathbf{r}}$ and $\tau_{\mathrm{L}}$ (the longitudinal relaxation time)
$\frac{\tau_{\mathrm{r}}}{\tau_{\mathrm{L}}}=\frac{U_{\mathrm{P}} M_{\infty}}{U_{\mathrm{S}} \Delta P_{\mathrm{V}(\max )}}$.

Taking the values of $U_{\mathrm{P}}, U_{\mathrm{s}}$, and $\Delta P_{\mathrm{V} \text { (max) }}$ (and bulk modulus $K=M_{\infty}$ ) from Rigden et al. (1988), $\tau_{\mathbf{r}} / \tau_{\mathbf{L}}$ decreases from $>300$ to $\cong 3$ with increasing pressure to $25 \mathrm{GPa}$ and decreases further to $\cong 2$ at pressures greater than $25 \mathrm{GPa}$ (i.e. with increasing pressure, the rise time approaches the relaxation time of the mell). Rigden et al. (1988) discussed the possibility of approaching the glass transition at high pressure but were unable provide the above estimate of $\tau_{\mathrm{r}} / \tau_{\mathrm{L}}$.

The present analysis, combined with the estimated width of the viscoelastic region (see above) indicates that the discontinuity in bulk modulus observed by Rigden et al. (1988) at $25 \mathrm{GPa}$ may well indicate the onset of unrelaxed behavior rather than equilibrium behavior (c.f., Angell et al. 1987, Rigden et al. 1988).

The effect of pressure on the position of the $\tau$ curves, can be generally estimated from the pressure-dependence of viscosity. Thus, in Figure 3 , the $\tau$ curves of relatively depolymerized melts such as diopside are expected to rise with pressure whereas the $\tau$ curves of polymerized melts such as albite are expected to lower with pressure (Scarfe et al. 1987). This has significance for modeling volume relaxation of densified glass. Höfler and Seifert (1984) have performed volume relaxation experiments on densified $\mathrm{SiO}_{2}$ by annealing samples at high temperature and $1 \mathrm{~atm}$. Although the relaxation time experiments are performed at $1 \mathrm{~atm}$, the state of the $\mathrm{SiO}_{2}$ preserves a high pressure memory via its densification. Thus the $\tau$ curve of Figure 3 for $\mathrm{SiO}_{2}$ must be corrected for pressure before comparison with the Höfler and Seifert (1984) data. Their data for the relaxation time of the volume relaxation process are presented in Figure 3 where it is apparent that they fall below the low temperature extrapolation of the 1 atm $\tau$ curve for $\mathrm{SiO}_{2}$. If we estimate the effect of pressure on the viscosity of $\mathrm{SiO}_{2}$ (using $\mathrm{GeO}_{2}$ data from Scarfe et al. 1987) then we predict a lowering of the $\tau$ curve for $\mathrm{SiO}_{2}$ with increasing pressure. The $2 \mathrm{GPa}$ curve is plotted in Figure 3 as a dashed line which agrees well with the data of Höfler and Seifert (1984).

A significant conclusion can be drawn from the above. We have noted that each type of experiment has a characteristic timescale of observation and thus the invariance of $G_{\infty}$ yields a viscosity which defines the glass transition for each experiment. In practice, the important criterion is the onset of significant unrelaxed response which may be modeled by adding a $\log _{10}$ value of 2 to the viscosity calculated from Equation 8. When a sample has a viscosity lower than what may be termed the "transition" viscosity for the measurement technique, it will behave as a liquid in the experiment; when its viscosity is higher it will be unrelaxed, exhibiting viscoelastic or elastic properties. Thus if one wishes to obtain relaxed thermodynamic data on a particular sample one must choose a temperature that will result in a sample viscosity below the transition viscosity.

\section{Geological Strain Rates: Liquid versus Glassy Behavior in Magmatic Processes}

The relationship between temperature, magma composition and strain rates in nature is complex. Here we propose an outline of the relationship between the liquid-glass transition of typical natural silicate melt compositions and the 


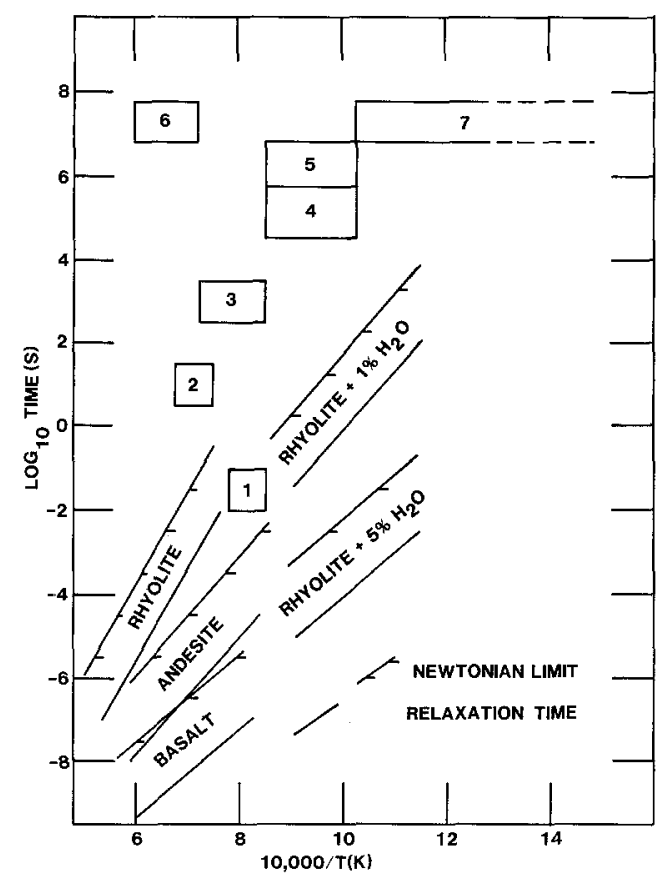

Fig. 4. The relaxation time and Newtonian limit of shear viscosity for common magma chemistry. The curves are derived from shear viscosities calculated using the method of Shaw (1972). The compositions are the R1A rhyolite of Spera et al. (1988) and the Crater Lake Andesite and Hawaiian Tholeiite of Tyburzcy and Waff (1983). The magmatic processes represented by boxes numbered 1-7 are 1 - ash flow eruption, 2 - basalt lava flow, 3 - andesite lava flow, 4 - rhyolite flow, 5 - silicic dome extrusion, 6 - basaltic melt segregation, 7 - granitic melt segregation. Strain rate estimates are from Spera et al. (1988)

strain rates operating during the magmatic processes of melt formation, ascent and emplacement or eruption. Figure 4 contains the $\tau$ curves derived for a rhyolite (dry and wet), an andesite and a basalt using viscosities calculated with the method of Shaw (1972). Various geological processes are located in terms of their temperatures and strain rates.

Recalling that the onset of non-Newtonian behavior is predicted to be approximately $2 \log _{10}$ units of time above each $\tau$ curve, it can be seen than dry rhyolitic melts can be expected to exhibit non-Newtonian behavior for processes such as ash flow eruptions and silicic dome extrusion. The effect of water on rhyolite will be significant but the lower temperatures associated with wet rhyolites and granites indicate that processes in wet silicic systems such as magmatic migmatite formation and melt segregation might result in viscoelastic (unrelaxed) behavior for anatectic melts. The andesite $\tau$ curve will be similarly affected by water and the behavior of andesite melt with a few percent water might be expected to be Newtonian for processes such as lava flow but non-Newtonian during violent eruptions. The $\tau$ curve for basalt appears to indicate that basaltic liquids will be Newtonian for most imaginable processes involving liquids of their chemistry such as lava flow or mantle segregation.

The effect of pressure on viscosity results in a convergence of the curves of Figures 3 and 4, at least up to $2.5 \mathrm{GPa}$ (Scarfe et al. 1987). Thus in Figures 3 and 4 the position of the $\tau$ curve becomes slightly less composition-dependent at these moderate pressures. In general rhyolite viscosity decreases strongly while andesite and basalt viscosities de- crease less strongly, with pressure, and their $\tau$ curves will lower accordingly in Figures 3 and 4.

\section{The Sheared State}

If, during a particular petrogenetic process, a silicate melt is located within 2 orders of magnitude of its $\tau$ curve (or lower in Figure 3) then the thermodynamic state of the melt will be strain-rate dependent. In fact strain-induced anisotropy is commonly observed as optical birefringence in the glass industry (Brückner 1987). Stockhorst and Brückner (1982) report optical birefringence in E-glass fibers drawn across the glass transition. The birefringence is a linear measure of optical anisotropy and the maximum reported value is 0.48 percent (Stockhorst and Brückner 1982). Attempts to characterize such anisotropy by orientational ${ }^{29} \mathrm{Si}$ NMR have produced a negative result (Stebbins and Farnan 1988). In this regard we suggest that the prediction of the preservation of such anisotropy requires a time-temperature-transformation (TTT) type of analysis of Figure 3.

The strain-rate dependence of intensive properties applies not only the intensive state variables of the system that we normally term physical properties (e.g., as viscosity, density, surface tension) but also to the intensive chemical properties such as the chemical potentials of components. One way to envisage the effect of strain-rate dependence of melt properties is in terms of a steady state where a proportion of the atoms in the system are held in an activated state relative to their thermal equilibrium. In the case of shear this could mean an increase in the proportion of atoms that exceed the activation energy of the diffusive oxygen jump. In turn, the activities of mineral components are presumably consequences of the proportion, distribution and linkage of silicate tetrahedra and thus changing any of these could affect mineral activities and liquid-crystal phase equilibria. Finally, we know that the solubilities of volatiles in silicate melts are composition-dependent, presumably reflecting changes in the activities of melt components. Thus shear-induced changes in component activities could affect volatile solubilities.

\section{Summary}

The relative invariance of $G_{\infty}$ with changes in composition and temperature allows the timescale of shear relaxation to be calculated for any silicate melt for which shear viscosity data are available. The results of such calculations yield a family of relaxation time or $\tau$ curves that describe the temperature-dependence of shear relaxation time for a range silicate melts. The calculated positions of the $\tau$ curves are consistent with evidence for relaxed versus unrelaxed behavior from a variety of studies (i.e. ultrasonics, mechanical torsion, fiber elongation, concentric cylinder viscometry, shock wave, volume relaxation, ${ }^{29} \mathrm{Si}$ NMR). Moreover, the calculated timescale of viscous relaxation corresponds to the timescale of a diffusive jump of oxygen in the melt.

The $\tau$ curves record the timescale of observation that corresponds to the liquid-glass transition for each composition, as a function of temperature. The high-temperature, long-timescale and the low-temperature, short-timescale regimes refer to liquid and glassy behavior, respectively. In the liquid state, all properties are strain-rate (or frequency) independent. In the glass regime, the energy input per unit strain is large enough to alter the thermodynamic state of 
the melt and intensive properties are strain-rate dependent. One example is the observation of non-Newtonian shear viscosity. Estimates of observation times in experiments allow prediction of whether relaxed or unrelaxed properties will be observed. Similarly, estimates of strain rates in magmatic processes allow the prediction of liquid versus glassy behavior in igneous petrogenesis.

Acknowledgements. We wish to thank M. Carroll, R. Luth, L. Merwin, A. Sebald and D. Virgo for discussions of this work. Helpful reviews were provided by P. Richet, J. Stebbins and E. Stolper. We wish to thank Fritz Seifert and the Bayerisches Geoinstitut for bringing together experimental geochemists and geophysicists to address problems of geological relevance.

\section{References}

Angell CA (1984) Strong and fragile liquids. Relaxations in Complex Systems. Ngai KL and Wright GB (eds) Office of Naval Research and National Technical Information Service

Angell CA, Torell LM (1983) Short time structural relaxation processes in liquids: comparison of experimental and computer simulation glass transition on picosecond timescales. J Chem Phys 78:937-945

Angell CA, Cheesemen PA, Kadiyala RR (1987) Diffusivity and thermodynamic properties of diopside and jadeite melts by computer simulation studies. Chem Geol 62:83-92

Astin AV (1962) Certificate of viscosity values. Standard sample No. 710 Soda-Lime-Silica Glass. US Dept of Commerce, Natl Bur Stds. Washington DC

Bansal NP, Doremus RH (1986) Handbook of glass properties. Academic Press, New York, London, pp 680

Bird RB, Armstrong RC, Hassager O (1977) Dynamics of polymeric liquids Vol 1. Wiley and Sons, New York, pp 470

Bucaro JA, Dardy HD (1974) High-temperature Brillouin scattering in fused quartz. J Appl Phys 45:5324-5329

Brückner R (1987) Structural aspects of highly deformed melts. J Non-Cryst Sol 95-96:961-968

Calas G, Hawthorne FC (1988) Introduction to spectroscopic methods. In: FC Hawthorne (ed) Spectroscopic methods in mineralogy and geology. Mineralogical Society of America Reviews in Mineralogy 18, 1-9

Dingwell DB, Scarfe CM, Cronin D (1985) The effect of fluorine on viscosities in the system $\mathrm{Na}_{2} \mathrm{O}-\mathrm{Al}_{2} \mathrm{O}_{3}-\mathrm{SiO}_{2}$ : implications for phonolites, trachytes and rhyolites. Am Mineral 70:80-87

Dunn T (1982) Oxygen diffusion in three silicate melts along the join diopside-anorthite. Geochim Cosmochim Acta 46:22932299

Glasstone S, Laidler KJ, Eyring H (1941) The theory of rate processes. McGraw-Hill, New York, pp 611

Gruber GJ, Litovitz TA (1964) Shear and structural relaxation in molten Zinc Chloride. J Chem Phys. 40:13-26

Herzfeld KF, Litovitz TA (1956) Absorption and dispersion of ultrasonic waves. Academic Press, New York, pp 535

Höfler S, Seifert FA (1984) Volume relaxation of compacted glass: a model for the conservation of natural diaplectic glasses. Earth Planet Sci Lett 67:433-438

Hofmaier G, Urbain G (1968) The viscosity of pure silica. Sci Ceram 4:25-32

Jackson I (1986) The laboratory study of seismic wave attenuation. In Mineral and rock deformation: Laboratory studies. (eds) Hobbs BE and Heard HC, 11-24, AGU

Kurkjian CR (1963) Relaxation of torsional stress in the transformation range of a soda-lime-silica glass. Phys Chem Glass $4: 128-136$

Laberge NL, Vasilescu VV, Montrose CJ, Macedo PB (1973) Equilibrium compressibilities and density fluctuations in $\mathrm{K}_{2} \mathrm{O}-\mathrm{SiO}_{2}$ glasses. J Am Cer Soc 56:506-509

Lambert JB, Nienhuis RJ, Keepers JW (1981) Kinetik intramoleku- larer Reaktionen aus Relaxationszeitmessungen. Angew Chem 93, 533-566

Lange RA, Carmichael ISE (1987) Densities of $\mathrm{Na}_{2} \mathrm{O}-\mathrm{K}_{2} \mathrm{O}$ $-\mathrm{CaO}-\mathrm{MgO}-\mathrm{FeO} \cdot \mathrm{Fe}_{2} \mathrm{O}_{3}-\mathrm{Al}_{2} \mathrm{O}_{3}-\mathrm{TiO}_{2}-\mathrm{SiO}_{2}$ liquids: New measurements and derived partial molar properties. Geochim Cosmochim Acta 51:2931-2946

Larsen DC, Mills JJ, Sievert JL (1974) Stress relaxation behavior of soda-lime glass between the transformation and softening temperatures. J Non-Cryst Sol 14:269-279

Li JH, Uhlmann DR (1970) The flow of glass at high stress levels. J Non-Cryst Sol 33:235-248

Litovitz TA, Davis CM (1965) Structural and shear relaxation in liquids. In: Mason WP (ed) Physical Acoustics Vol. I IA, Academic Press, New York, 281-349

Liu S-B, Stebbins JF, Schneider E, Pines A (1988) Diffusive motion in alkali silicate melts: an NMR study at high temperature. Geochim Cosmochim Acta 52:527-538

Mills JJ (1974) Low frequency storage and loss moduli of soda silica glasses in the transformation range. J Non-Cryst Solids $14: 255-268$

Muehlenbachs K, Schaeffer HA (1977) Oxygen diffusion in vitreous silica-utilization of natural isotopic abundances. Can Mineral 15:179-184

Nowick AS, Berry BS (1973) Anelastic relaxation in solids. Academic Press, New York, pp 677

Nye JF (1957) Physical properties of crystals. Oxford Press, Oxford, pp 322

O'Connell RJ, Budiansky B (1978) Measures of dissipation in viscoelastic media. Greophys Res Lett 5:5-8

Richet P (1984) Viscosity and configurational entropy of silicate melts. Geochim Cosmochim Acta 48:471-483

Rigden SM, Ahrens TJ, Stolper EM (1988) Shock compression of molten silicate: results for a model basaltic composition. $J$ Geophys Res 93:367-382

Rosen SL (1982) Fundamental principles of polymeric materials. Wiley and Sons, New York, pp 346

Ritland HN (1954) Density phenomena in the transformation range of a borosilicate crown glass. J Am Cer Soc 37:370-378

Rai CS, Manghnani MH, Katahara KW (1981) Ultrasonic studies on a basalt melt. Geophys Res Lett 8:1215-1218

Rivers ML, Carmichael ISE (1987) Ultrasonic studies of silicate melts. J Geophys Res 92:9247-9270

Ryan MP, Blevins JYK (1987) The viscosity of synthetic and natural silicate melts and glasses at high temperatures and 1 bar $\left(10^{5}\right.$ pascals) pressure and higher pressures. USGS Bull 1764, pp 563

Sato $H$, Manghnani MH (1985) Ultrasonic measurements of $V_{P}$ and $\mathrm{Q}_{\mathrm{p}}$ : relaxation spectrum of complex modulus on basalt melts. Phys Earth Planet Int 41:18-33

Scarfe CM, Cronin DJ, Wenzel JT, Kauffman DA (1983) Viscositytemperature relationships at $1 \mathrm{~atm}$ in the system diopside-anorthite. Am Mineral 68:1083-1088

Scarfe CM, Mysen BO, Virgo D (1987) Pressure dependence of the viscosity of silicate melts. Magmatic Processes: Physicochemical Principles, Mysen BO (ed) 59-67

Shaw HR (1972) Viscosities and magmatic silicate liquids: an empirical method of prediction. Am J Sci 272:870-893

Shimizu N, Kushiro I (1984) Diffusivity of oxygen in jadeite and diopside melts at high pressures. Geochim Cosmochim Acta 48:1295-1303

Simmons JH, Mohr RK, Montrose CJ (1982) Non-Newtonian viscous flow in glass. $\mathrm{J}$ Appl Phys 53:4075 4080

Spera FJ, Borgia A, Strimple J, Feigenson M (1988) Rheology of melts and magmatic suspensions. I. Design and calibration of concentric cylinder viscometer with application to rhyolitic magma. J Geophys Res 93:10273-10294

Stebbins JF, Farnan I (1988) Spatial orientation of structural units in silicate glasses: results from NMR spectroscopy. EOS 69 , 504 (abstr)

Stockhorst H, Brückner R (1982) Structure sensitive measurements on E-glass fibers. J Non-Cryst Sol 49, 471-484 
Sucov EW (1963) Diffusion of oxygen in vitreous silica. J Am Cer Soc $46: 14-20$

Tauke J, Litovitz TA, Macedo PB (1968) Viscous relaxation and non-Arrhenius behavior in $\mathrm{B}_{2} \mathrm{O}_{3}$. J Am Ceram Soc 51:158-163

Tyburzcy JA, Waff HS (1983) Electrical conductivity of molten basalt and andesite to 25 kilobars pressure: geophysical significance and implications for charge transport and melt structure. J Geophys Res 88:2413-2430

Weast RC (1972) Handbook of physics and chemistry, CRC Press, Cleveland, pp 2335
Williams EL (1965) Diffusion of oxygen in fused silica. J Am Cer Soc 48: 190-194

Woodcock LV, Angell CA, Cheeseman P (1976) Molecular dynamic studies of the vitreous state: simple ionic systems and silica. J Chem Phys 65:1565-1577

Received November 28,1988 